\title{
Perspectives on behavior and acquired brain injury
}

\author{
Harvey E. Jacobs*
}

Behavior!

When most people associate behavior with acquired brain injury (ABI) they identify problems; problems ranging from aggression and agitation to non-compliance and depression (Baguley, Cooper, Felmingham, 2006; Jorge, Robinson, Moser, Tateno, Crespo-Facorro, Arndt, 2004; Kim, 2002). Requested treatment goals often focus on the cessation of aberrant actions on the part of the individual, frequently using a selected armamentarium of consequence based procedures or medication. Not infrequently, staff or family members may exasperate that "we've tried everything else, so I guess we're left with behavioral procedures!" Per everybody's hope, the person of focus, typically the person who experiences disability following ABI, will ultimately "get it," heals, or achieves a new homeostasis. This person also often has only a limited say in developing treatment plans.

However, behavior is not limited to unsanctioned actions. It constitutes all of our actions and representations to others - the interaction of an organism (person) with their environment. "Behavior" includes competencies as well as incompentencies, function as well as dysfunction, and expands beyond classical behavioral paradigms. A promising literature has developed regarding facilitation of individual capacity in light of notable neurological, cognitive, emotional and behavioral impairment (Ylvisaker and Feeney, 1998; Ylvisaker, Jacobs, Feeney, 2003). Not surprisingly, these proactive approaches also often reduce or

*Address for correspondence: Harvey E. Jacobs, Richmond, VA, USA. E-mail: harveyjacobs@ harveyjacobs.net. eliminate aberrant situations (Gardner, Bird, Maguire, Carreiro, Rui, Abenaim, 2003). Simply and colloquially speaking, most people want to succeed.

Behavior dysfunction, according to many of these approaches may be best construed as a sentinel than a cause. It signals that a person is beyond his or her personal capacities and needs contextually relevant supports to approach presenting challenges. These supports often transcend traditional/clinical models of treatment and recognize holistic aspects of the individual and their circles of support, especially those providing services (Cattelain, Zettin, Zoccolotti, 2010).

The purpose of this special issue is to move beyond the person and the brain. The scientific literature has adroitly documented the impact of neurological impairment on behavior, including noted effects on perception, executive function, awareness, self-management, memory, communication, comprehension and emotional control, among many other factors (Silver, McAllister, Yudofsky, 2011; Zasler, Katz, Zafonte, Arciniegas, Bullock, Kreutzer, 2012). With a loss of such capacity comes decreased personal locus of control and an increasing dependency, sensitivity and vulnerability to environmental factors. Equal focus is required to understand more clearly how our behavior, especially those involved in service delivery or caregiving, directly or through our systems, diagnostic and treatment perspectives, cultures and perceptions directly affects behavior associated with ABI, with an emphasis on competence over dysfunction (Jacobs, 2010).

The first article by Buzan et al. truly expands concepts and definitions of behavior. The authors note 
that individuals with brain injury and their families frequently struggle to accept post-injury personality changes. This process is complicated by tacit assumptions regarding the nature of personality, free will, and the relationship between the mind and the brain. The authors challenge the Western Dualistic model of mind and body by reviewing the constructional nature of perception, and the neurologic bases of affect, morality, empathy, and sense of self. The sense of self, while conflated with the concept of a "soul" in Western thinking, is more rationally considered as a construct derived from neurophysiologic structures. The authors discuss embodiment theory as one viable solution to the mindbody dilemma.

As noted by Wortzel and Arciniegas, the Diagnostic and Statistical Manual of Mental Disorders, Fifth Edition (DSM-5) invokes substantial changes regarding diagnosis of traumatic brain injury (TBI) and its neuropsychiatric sequelae. This in turn is likely to affect treatment decisions. TBI is now primarily discussed as a Mild or Major Neurocognitive Disorder. Diagnostic determination is now based retrospectively on the severity of posttraumatic cognitive impairments and their effects on everyday function, as compared to the initial severity of TBI. The authors conclude that the DSM5 is likely to improve the evaluations of persons with TBI by mental health professionals, including differential diagnosis, and that experience with DSM-5 is likely to improve DSM-5.1!

Multiple factors contribute to post-injury dysfunction. Webb et al. outlines common neuroendocrine disruptions secondary to TBI along with their clinical implications. The authors note that TBI initiates a cascade of neuromodulatory damage that blurs distinctions between physical and psychological medicine. Monitoring endocrine function is critical to avoid misdiagnosing and mistreating clinical symptoms such as depression, fatigue, diminished concentration, irritability and overall cognitive decline. Wider adoption of consensus guidelines on the detection and monitoring of endocrine abnormalities post-TBI may diminish the severity of functional impairment and improve quality of life.

McQueen and O'Shanick note that acute rehabilitation following neurotrauma has evolved over the past 30 years to include the common use of pharmacologic agents to promote synaptogenesis and improve recovery potential. However, little guidance exists for similar strategies in post-acute or community re-entry phases of ABI. Drawing upon the existing scientific literature, models of pharmacologic intervention in promoting stability in other disease states and the authors' collective clinical experience, this article provides a potential structure to create a stable physiologic platform to facilitate pro-active behavioral intervention.

Lequerica and Krch explore how issues of cultural diversity and staff perceptions affect treatment outcomes. As the general population in the United States becomes increasingly diverse, it becomes more important to provide culturally sensitive treatment. This is especially relevant when working with minority populations who have a higher risk for brain injury and poorer rehabilitation outcomes. Being able to regard behavior after brain injury with an open mind and understanding one's own ethnocentrism relative to cultural and contextual factors are important steps in developing competent rehabilitation practices and moderating adverse behavioral sequelae.

Karol provides perspectives on team models, in particular the advantages of trans-disciplinary teams compared to multi or inter-disciplinary approaches in neurorehabilitation treatment. The paper highlights the roles that team members play in the different team models and associated effects on care coordination and outcomes. The concepts of role release; letting other disciplines practice in one's own traditional arena; and expanded scope of practice are explored. Crucial issues in the implementation of trans-disciplinary teams including, staff selection, rounds attendance, physical plant, peer pressure, and culture change are also discussed. An example of leadership introducing the trans-disciplinary model highlights these issues.

LeBel et al. provides an overview of work conducted to reduce the use of coercion, restraint, seclusion and other invasive practices in mental health settings that often include the defacto admission of persons with ABI. The article examines treatment culture factors that can exacerbate behavior dysfunction, how to moderate such challenges and prevent the use of coercive and restrictive procedures. When these procedures are recognized as an inadequate organizational response to harmful behavior that maintains patterns of aggression or harm, leadership-driven strategies, such as the Six Core Strategies ${ }^{\circledR}$ can provide a prevention based framework to anticipate challenges, intervene early, and disrupt the behavioral sequence leading to the use of coercive and restrictive procedures.

Highlighting one approach to facilitate personal competence, Jackson et al. explores key elements of structure in post-acute brain injury rehabilitation that can promote greater levels of personal functioning, emotional and behavioral stability and independence. 
Through careful clinical management and integration of environmental factors, therapeutic alliances, organizational structures and rehabilitation practices, many people with neurobehavioral challenges can transition from externally supported programmatic structures to processes of "self-structuring" that promote greater independence and quality of life. Achieving such outcomes requires robust infrastructures that can flexibly adapt to each person's needs and capacities.

In a follow up article, Jackson et al. report preliminary results of a standardized instrument to assess self-structuring. The Behavioural Assessment of SelfStructuring (BASS) is a 26 item, staff rating scale that evaluates individual client capacity to achieve selfstructuring. Initial results indicate that the BASS has reasonably good reliability, good construct validity, good discriminant validity, good concurrent validity and sensitivity to change. Additional work is in progress to further substantiate its psychometric properties. However, initial results are encouraging in assessing rehabilitation progress, especially as it relates to concepts of self-structuring.

Feeney and Achilich demonstrate the use of multi-component behavioral interventions to address challenging behaviors within natural settings. The two reported interventions extended the findings of previously published data for the effective treatment of behavioral challenges using a flexible support-oriented intervention that combines behavioral, cognitive, and executive function components. Though identifying such people in the title as "Chronically Cranky" may sound pejorative, the intent of the authors is to reflect how others often view such individuals, which in turn can affect treatment considerations unless one considers a holistic perspective of each situation.
In closing, I would like to extend my gratitude to the authors who so unselfishly contributed to this special issue. Your time and knowledge are so greatly appreciated.

\section{References}

Baguley, I.J., Cooper, J., \& Felmingham, K. (2006). Aggressive behavior following traumatic brain injury: How common is common? J Head Trauma Rehab, 21(4), 45-56.

Cattelain, R., Zettin, M., \& Zoccolotti, P. (2010). Rehabilitation treatments for adult with behavioral and psychosocial disorders following acquired brain injury: A systematic review. Neuropsychol Rev, 20, 52-85.

Gardner, R.M., Bird, F.L., Maguire, H., Carreiro, R., \& Abenaim N. (2003). Intensive positive behavior supports for adolescents with acquired brain injury: Long-term outcomes in community settings. J Head Trauma Rehab, 18(1), 52-74.

Jacobs, H.E. Understanding Everybody's Behavior After Brain Injury: Don't "Don't!"(T) (2010). Originally Youngsville, NC, US: Lash and Associates. Now available through author.

Jorge, R.E., Robinson, R.G., Moser, D., Tateno, A., Crespo-Facorro, B., \& Arndt, S. (2004). Major depression following traumatic brain injury. Arch Gen Psychiatry, 61(1), 42-50.

Kim, E. (2002). Agitation, aggression, and disinhibition syndromes after traumatic brain injury. Neurorehabilitation, 17(4), 297-310.

Silver, J.M., McAllister, T.W., \& Yudofsky, S.C. (Eds.). (2011). Textbook of Traumatic Brain Injury: Second Edition. Arlington, VA, US: American Psychiatric Publishing, Inc.

Ylvisaker, M., \& Feeney, T.J. (1998). Collaborative Brain Injury Intervention: Positive Everyday Routines. San Diego, CA, US: Singular Publishing Group.

Ylvisaker, M., Jacobs, H.E., \& Feeney, T. (2003). Positive supports for people who experience behavioral and cognitive disability after brain injury: A review. J Head Trauma Rehab, 18(1), 7-32.

Zasler, N.D., Katz, D.I., Zafonte, R.D., Arciniegas, D.B., Bullock, M.R., \& Kreutzer, J.S. (Eds.). (2012). Brain Injury Medicine, 2nd Edition: Principles and Practice. New York, NY, US: Demos Medical. 